(2) Open Access Full Text Article

ORIGINAL RESEARCH

\title{
Age and sex differences in neural stem cell transplantation: a descriptive study in rats
}

This article was published in the following Dove Press journal:

Stem Cells and Cloning:Advances and Applications

8 April 201I

Number of times this article has been viewed

Jay Waldron'

Laurent Lecanu ${ }^{1,2}$

'Research Institute of the McGill University Health Centre, Montreal, QC, Canada; ${ }^{2}$ McGill University

Health Centre, Department of

Medicine, QC, Canada
Correspondence: Laurent Lecanu

Research Institute of the McGill

University Health Centre, Royal Victoria

Hospital, Room L2-05, 687 Pine Avenue

West, Montreal H3A IAI, QC, Canada

$\mathrm{Tel}+\mathrm{I} 5142458180$

$\mathrm{Fax}+|5| 42836282$

Email laurent.lecanu@mcgill.ca
Purpose: The purpose of this study was to determine whether neural stem cell (NSC) sexual dimorphism previously demonstrated in vitro translates in vivo in NSC transplantation experiments and constitutes a defining factor of the transplantation outcome.

Methods: NSCs isolated from the subventricular zone of 2-day-old or 20-month-old male and female rats were grown as neurospheres prior to being transplanted in the striatum of 2-day-old or 20-month-old male and female recipient animals. The outcome of the transplantation and the NSC differentiation status were analyzed 8 weeks later by assessing the expression of the markers doublecortin (DCX) for neuroblasts, glial fibrillary acidic protein (GFAP) for astrocytes, nestin for stem cells, and choline acetyltransferase (ChAT) for neuronal cholinergic phenotype by immunofluorescence.

Results: No NSCs were detected in the brain of rat pups 8 weeks after transplantation. However, the endogenous neurogenesis was dramatically increased in a sex-dependent manner. These data suggest that the transplanted NSCs may have triggered endogenous neurogenesis by the intermediate growth factors they may have produced or the production they may have induced. However, NSCs transplanted into the striatum of adult rats were detectable at week 8. NSC survival was dependent on the sex and age of the donor and the recipient. Some of the transplanted cells were found to express DCX, GFAP, and ChAT, supporting an ongoing differentiation process toward astroglial and neuronal cholinergic phenotypes.

Conclusion: The outcome of the NSC transplantation was highly dependent on the sex and age of the combination donor/recipient. Data generated from our work may allow us in the future to answer the question "What NSCs and for whom?" and consequently lead to the optimization of the grafting process and improvement of the clinical prognosis.

Keywords: sexual dimorphism, neurogenesis, brain repair, stem cell graft

\section{Introduction}

Stem cell therapy for tissue regeneration and functional recovery is a main avenue of brain repair strategy. The controversy raised by the use of human embryonic stem cells and the discrepancy observed in the outcome of mesenchymal cell transplantation seem to be major setbacks to significant clinical development. In these conditions, neural stem cells (NSCs) seem to offer an interesting alternative with one main advantage of originating from the same organ into which they are meant to be grafted.

Endogenous NSCs have been shown to be nurtured in a specific "niche" microenvironment that provides specific cell-cell interaction required for the regulation of NSC proliferation and differentiation. This nursing structure does not exist when exogenous NSCs are grafted into a brain structure. Therefore, it is critical 
to determine the fate of NSCs that are transplanted in such a challenging environment. We recently demonstrated that NSCs differentially express estrogen receptors (ERs) and aromatase depending on the donor's sex and age. ${ }^{1,2}$ This sex dimorphism might account for the differential neural fate followed by these NSCs upon stimulation with the differentiating agent retinoic acid. ${ }^{1}$ Sex dimorphism between males and females has been demonstrated to occur in muscle-derived stem cells and bone marrow stem cells and to reflect on the organs' physiology. ${ }^{3-7}$ These results led us to believe that the sexual dimorphism we have evidenced in NSCs might translate into a differential fate after transplantation or even into a differential reaction of the recipient brain, depending on the sex and age of the NSC donor.

Herein we present a descriptive and comparative analysis of the outcomes of grafting male or female NSCs isolated from the subventricular zone (SVZ) of 2-day-old or 20-month-old rats in the striatum of male and female 2-day-old or 20-month-old recipients.

\section{Materials and methods}

DMEM/F12, Leibovitz L15 medium, B27, Neurobasal A, EGF, penicillin/streptomycin, PBS1X, PBS10X, gentamycin, and DNAse I were from Invitrogen (Carlsbad, CA); papain, poly-L-ornithine, laminin, and heparin were from Sigma (St Louis, MO); Percoll ${ }^{\circledR}$ and density marker beads were from GE Healthcare (Uppsala, Sweden); bFGF was from PeproTech (Rocky Hill, NJ); L-glutamine was from Thermo Fisher Scientific (Waltham, MA); Accumax ${ }^{\circledR}$ was from Innovative Cell Tech (San Diego, CA); glass-bottom chamber slides were from Nunc (Rochester, NY); and lowattachment six-well plates were from Corning (Avon, France). The primary antibodies used were specifically doublecortin and nestin (Cell Signaling Technology, Pickering, ON) and choline acetyltransferase (Millipore, Temecula, CA). Secondary antibodies DyLight 488 and DyLight 594 were purchased from Jackson Immunoresearch (West Grove, PA).

\section{NSC isolation and amplification}

NSCs were isolated for rat SVZ as previously described. ${ }^{1,2}$ NSCs were not pooled after isolation, and each animal provided its own NSC batch. Male and female 2-day-old and 20-monthold Long-Evans rats were anesthetized with 3\% isoflurane and transcardiacally perfused with DMEM/F12 medium (only adult animals). Following decapitation, the brains were removed and rinsed in phosphate buffered saline (PBS)/1\% penicillin/streptomycin/gentamycin (PSG) before being placed in sterile plastic Petri dishes containing Leibovitz
L15 medium on ice. Two coronal cuts were made in the area between the rhinal fissure and the hippocampus. The resulting tissue chunk was placed on its posterior surface and the cortex above the corpus callosum and surrounding the ventricles removed. ${ }^{8}$ The remaining tissue (SVZ) was placed in another sterile Petri dish containing L15 with $2.5 \mathrm{UI} / \mathrm{mL}$ papain, $10 \mathrm{UI} / \mathrm{mL}$ DNAse I, and 1\% PSG and diced into approximately $1 \mathrm{~mm}^{3}$. The diced SVZ were each transferred to $50 \mathrm{~mL}$ centrifuge tubes containing $25 \mathrm{~mL}$ L15 media plus $2.5 \mathrm{UI} / \mathrm{mL}$ papain, $10 \mathrm{UI} / \mathrm{mL}$ DNAse I, and $1 \%$ PSG and allowed to digest for 40 minutes at $37^{\circ} \mathrm{C}$ in a mixer incubator. Following digestion, the tissue suspensions were centrifugated at $300 \mathrm{rpm}$ for 2 minutes and pellets were resuspended in $25 \mathrm{~mL}$ DMEM/F12 containing $10 \mathrm{UI} / \mathrm{mL}$ DNAse, 2\% B27, and 1\% PSG and centrifuged at $300 \mathrm{rpm}$ for another 2 minutes. The supernatants were removed and replaced with $3 \mathrm{~mL} 37^{\circ} \mathrm{C} \mathrm{DMEM} / \mathrm{F} 12$. Following trituration with a disposable pipette, the homogenates were passed sequentially through $100 \mu \mathrm{m}, 70 \mu \mathrm{m}$, and $40 \mu \mathrm{m}$ cell strainers. The filtrated homogenates were then transferred to a $10 \mathrm{~mL}$ centrifuge tube, and the volume was increased to 4.5 mL with DMEM/F12 to which was added $4.5 \mathrm{~mL}$ Percoll solution (Percoll:PBS 10X 9:1). This mixture was centrifuged at $12,000 \mathrm{rpm}$ at $18^{\circ} \mathrm{C}$ for 1 hour. Density marker beads (GE Healthcare) were used to determine the layer corresponding to the NSCs $(1.065-1.075 \mathrm{~g} / \mathrm{mL}) .{ }^{9}$ These cells were placed in a sterile $15 \mathrm{~mL}$ centrifuge tube and rinsed with $15 \mathrm{~mL}$ of $37^{\circ} \mathrm{C} \mathrm{DMEM} / \mathrm{F} 12$. Following centrifugation for 10 minutes at $900 \mathrm{rpm}, 14.5 \mathrm{~mL}$ of supernatant was removed and replaced with $14.5 \mathrm{~mL}$ Neurobasal A at $37^{\circ} \mathrm{C}$ containing $2 \%$ B27, $2 \mathrm{nM}$ L-glutamine, $20 \mathrm{ng} / \mathrm{mL}$ EGF, $20 \mathrm{ng} / \mathrm{mL} \mathrm{bFGF}$, and $2 \mu \mathrm{g} / \mathrm{mL}$ heparin. The cells were centrifuged for 10 minutes at $900 \mathrm{rpm}$, and resuspended cells were seeded in six-well lowattachment plates and incubated at $37^{\circ} \mathrm{C}$. EGF, bFGF, and heparin were added to cells three times a week, and culture medium was changed once a week. Neurospheres were split every month using Accumax ${ }^{\circledR}$. NSCs from various animals were not pooled and used as a single animal source.

\section{Neurosphere transplantation}

All experimental protocols involving animals were approved by the Biotechnology Research Institute (National Research Council of Canada) Animal Care Committee and by the Animal Care Committee of the Research Institute of the McGill University Health Centre. Experiments were performed according to the code of practice for animal experimentation of the Animal Welfare Act and the Public Health Service Policy on Laboratory Animal Care. 
On the day of transplantation, neurospheres were gently dissociated in a single cell suspension using Accumax ${ }^{\circledR}$. NSCs were then labeled with the fluorescent marker Kodak $\mathrm{X}$-sight 650 Nanosphere ${ }^{\mathrm{TM}}$ (Carestream) (5 $\mathrm{MM}$ in the culture medium for 3 hours) prior to transplantation in order to track transplanted cells. NSCs were harvested and resuspended in Dulbecco's PBS containing bFGF $20 \mathrm{ng} / \mathrm{mL}$. Using stereotaxic injection, $5 \mu \mathrm{L}$ of the cell solution at a concentration of approximately 5000 cells $/ \mu \mathrm{L}$ was injected into the left striatum of deeply anesthetized male and female 2-day-old and 20-month-old rats. Both male and female 2-day-old and 20-month-old recipients received either male or female NSCs isolated from 2-day-old and 20-month-old rats. Each recipients group included four pups or four adult rats. Each recipient received NSCs derived from a different animal in order to minimize a potential "NSC batch" effect. After the transplantation procedure, the pups were returned to their respective mother until weaning. None of the grafted animals was immunosuppressed.

\section{Immunofluorescence}

\section{Brain tissue}

At week 8 after implantation, the rats were anesthetized with $3 \%$ isoflurane and transcardiacally perfused with sodium chloride $(\mathrm{NaCl}) 0.9 \%$ at $37^{\circ} \mathrm{C}$ until complete exsanguination followed by a perfusion of $4 \%$ paraformaldehyde. Fixed brains were then removed and stored at $-86^{\circ} \mathrm{C}$ until processing. Neural cells originating from transplanted NSCs were visualized by immunofluorescence and their phenotype characterized using antibodies raised against specific markers. A total of $30 \mu \mathrm{m}$-thick coronal sections were obtained using a cryostat (Leica CM3050 S). Immunostaining was performed on floating sections corresponding to the lateral ventricles/ SVZ/striatum area using primary antibodies specific to nestin, glial fibrillary acidic protein (GFAP), or DCX (24 hours at $4^{\circ} \mathrm{C}$ ). Secondary antibodies (DyLight 488 and DyLight 594) were incubated at $4^{\circ} \mathrm{C}$ for 24 hours.

\section{Neurospheres}

Primary neurospheres were pipetted into glass-bottom chamber slides (10-15 neurospheres per well) that were coated with poly-L-ornithine and laminin. Neurospheres were then maintained in the same medium as when amplified for a week in order to let them attach. Thereafter, neurospheres were washed in PBS $1 \mathrm{X}$ and fixed in methanol at $-20^{\circ} \mathrm{C}$ for 10 minutes. Immunolabeling was conducted using choline acetyltransferase (ChAT) primary antibody to assess the level of ChAT expression in undifferentiated NSC neurospheres.
Primary antibody was incubated 24 hours at $4^{\circ} \mathrm{C}$ followed by secondary antibody DyLight 488 in the same conditions. NSC nuclei were counterstained with 4',6-diamidino-2phenylindole (DAPI).

Brain sections and neurosphere immunofluorescence pictures were obtained using a FluoView 1000 confocal microscope (Olympus, Markham, ON).

\section{Results \\ NSC transplantation outcome in 2-day-old pups}

A common pattern to all the transplantations reported herein is that no grafted cells were detectable in any of the recipient animals analyzed at 8 weeks. However, we observed histological modifications and alteration of the endogenous expression of DCX and nestin in the SVZ of the grafted animals that seemed to depend on the sex and age of the rats from which NSCs were isolated. Overall, the transplantation of NSCs of a given sex/age resulted in histological modifications of the SVZ that were similar for male and female recipients.

Transplantation of male 2-day-old NSCs resulted in a significant modification of the SVZ morphology in both male and female recipients. Male ipsilateral SVZ displayed a cluster-like structure (Figure 1A) protruding inside the ventricle and expressed an increased amount of the neuroblast marker DCX and stem cell marker nestin (Figure 1A, 1A1, and 1A2) compared with the contralateral side (Figure 1B, 1B1, and 1B2). In addition, DAPI staining showed an increased number of cells and a thickening of the subependymal layer on the ipsilateral side. Female recipients' ipsilateral ependymal/subependymal structure also displayed morphological modification, although more dramatic than the one observed in males (Figure 1C). In addition, clusters of cells with increased DCX and nestin expression were observed (Figure 1C, 1C1, and 1C2, arrow). As for 2-day-old male NSCs, the transplantation of 2-day-old female NSCs resulted in a dramatic increase of DCX- and nestin-positive cells in the ipsilateral SVZ (Figure 1E and 1G, respectively) compared with the contralateral side (Figure 1F and 1H, respectively). However, unlike what was observed in the animals grafted with 2-day-old male NSCs, the rats grafted with 2-day-old female NSCs displayed a regular and homogenous thickening of the area where cells overexpressing DCX and nestin were present. In addition, the ependymal area closest to the corpus callosum on the ipsilateral side, in both male and female recipients, displayed an increased number of cells expressing DCX and nestin as compared with the contralateral side (Figure 1E-1H, 1E1-1H1, and 1E2-1H2). 


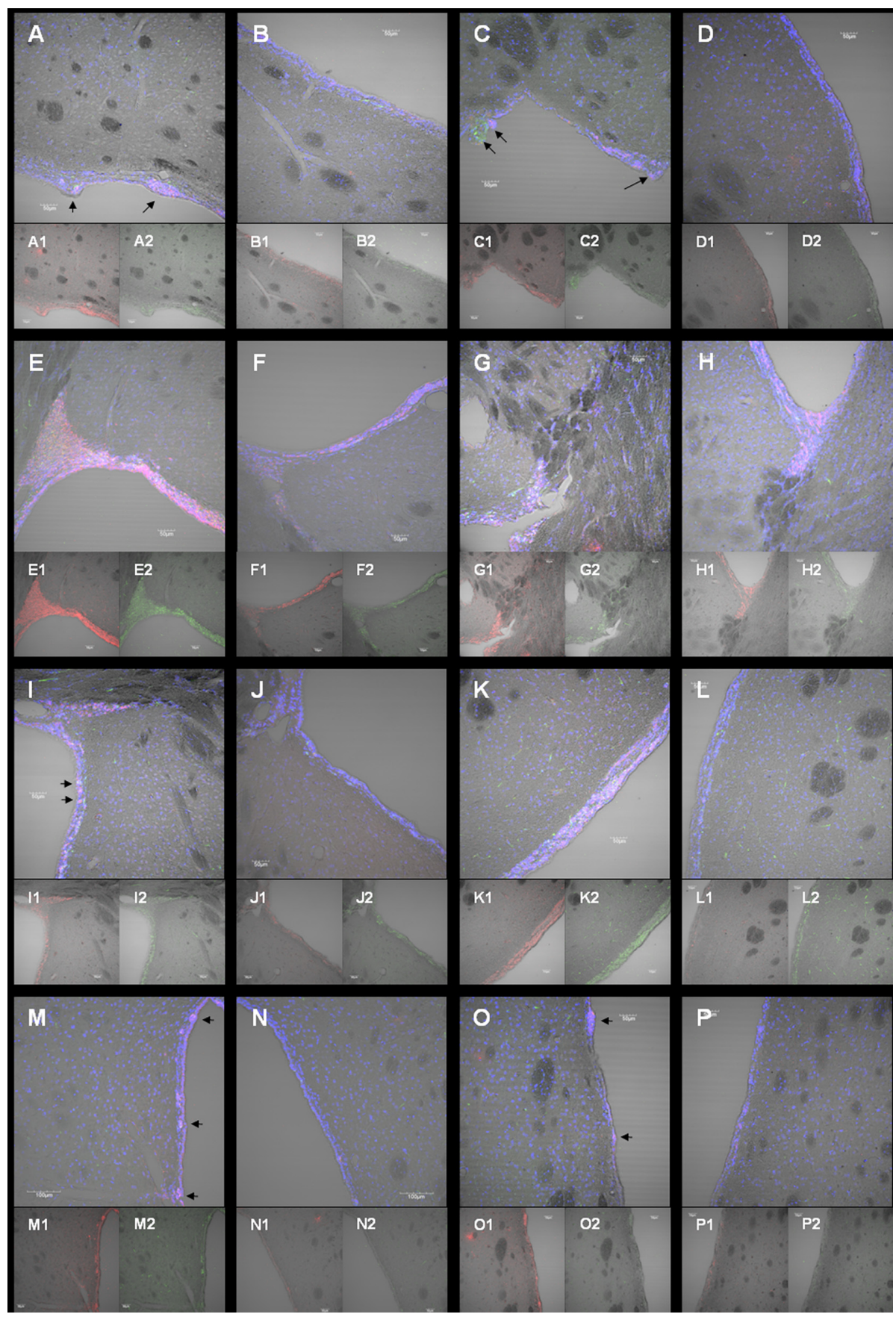

Figure I Neural stem cell (NSC) transplantation outcome in 2-day-old pups. Two-day-old pups, male and female, were grafted in the left striatum with male or female NSCs isolated from the subventricular zone of either 2-day-old or 20-month-old rats. NSCs were labeled with the fluorescent probe Kodak X-sight 650 Nanosphere ${ }^{\text {TM }}$. The outcome of the transplantation was then analyzed 8 weeks after the surgical procedure. Immunostaining was performed to specifically detect the neuroblast marker doublecortin (DCX) (A2-P2, green) and the stem cell marker nestin (AI-PI, red). Cell nuclei were visualized using the fluorophore 4',6-diamidino-2-phenylindole. Male recipients: ipsilateral (A, E, I, and M); contralateral (B, F, J, and N). Female recipients: ipsilateral (C, G, K, and O); contralateral (D, H, L, and P). Two-day-old male NSCs (A-D); 2-day-old female NSCs (E-H); 20-month-old male NSCs (I-L); 20-month-old female NSCs (M-P). (A-P) represent one single confocal plan, and AI/A2-PI/P2 represent the full stack for a better rendering. The black arrows indicate cell clusters expressing nestin and/or DCX.

The transplantation of 20-month-old male NSCs induced an increased expression of DCX and nestin in both male and female recipients. The ipsilateral SVZ displayed a higher number of nestin-positive and DCX-positive cell clusters (Figure 1I and 1K) compared with the contralateral side
(Figure $1 \mathrm{~J}$ and 1L). A slight thickening of the SVZ was also observed, which was probably due to a higher cell density, although this morphological alteration appeared to be more dramatic in female than in male recipients. Male and female rats grafted with 20-month-old female NSCs 
did not display a continuous and homogenous increase of the number of nestin- and DCX-positive cells in the SVZ but rather a few more cell clusters in the ipsilateral SVZ than in the contralateral SVZ (Figure $1 \mathrm{M}$ and $1 \mathrm{P}$ ). In addition, and unlike what was observed in the other groups, the transplantation of 20-month-old female NSCs did not result in the SVZ thickening.

\section{NSC transplantation outcome in 20-month-old rats}

Unlike what has been observed in the transplantations performed in 2-day-old pups, the NSCs grafted in 20-month-old animals could still be seen, in large numbers in some cases, 8 weeks after the surgical procedure.

\section{Expression of the neuroblast marker doublecortin and the astrocyte marker GFAP}

In 20-month-old male recipients, the most significant results in the term of NSC survival and neural marker expression were obtained with the transplantation of 20-month-old female NSCs (Figure 2D). Many grafted cells were visible in the striatum along the needle track but also at a significant distance ( $200 \mu \mathrm{m}$, yellow arrows), as shown by the detection of the Kodak X-sight 650 Nanosphere ${ }^{\text {TM }}$ fluorescence (Figure 2D1). These cells were also expressing the neuroblast marker DCX with immunostaining colocalized with the probe signal (Figure 2D and 2D2). The astrocyte marker GFAP was found to be expressed by the engrafted cells and also in their vicinity, probably by supporting endogenous astrocytes (Figure 2D and 2D3). Interestingly, same-sex grafting of 20-month-old male NSCs did not seem to lead to significant cell survival (Figure 2C, yellow arrows). Only very few grafted cells were observed (Figure 2C1) to be expressing DCX (Figure 2C2) and were all localized in brain microvessels, either in the lumen or seemingly integrated to the wall (Figure 2C3). The analysis of the outcome of 2-day-old NSC transplantation led to the exact opposite observation. The most significant result was obtained with the same-sex grafting of 2-day-old male NSCs (Figure 2A). Indeed, numerous grafted cells were visible (Figure 2A1) expressing DCX (Figure 2A2) or GFAP (Figure 2A3). Endogenous supporting astrocytes were also seen surrounding the grafted cells (Figure 2A3). Only very few grafted cells were detected after cross-sex transplantation of 2-day-old female NSCs (Figure 2B and 2B1). As in the case of the transplantation of 20-month-old NSCs, these cells were expressing DCX (Figure 2B2) and located in the lumen or in the wall of brain microvessels (Figure 2B, yellow arrows).

The outcome of NSC transplantation in 20-month-old female brains followed the same pattern observed in males. Same-sex grafting of 2-day-old NSCs (Figure 3B) and cross-sex grafting of 20-month-old NSCs (Figure 3C) resulted in the highest NSC survival rate. Nanosphere ${ }^{\mathrm{TM}}$ fluorescence-positive cells were visible in both cases in the striatum of grafted animals (Figure $3 \mathrm{~B} 1$ and $3 \mathrm{C} 1$, respectively). In addition, as previously observed with the male recipient, transplanted NSCs were expressing the neuroblast marker DCX (Figure 3B2 and 3C2, respectively) or GFAP (Figure 3B3 and 3C3, respectively) and were surrounded by numerous astrocytes. However, 8 weeks after the transplantation, few cells were observed in the case of cross-sex grafting of 2-day-old NSCs (Figure 3A) and samesex grafting of 20-month-old NSCs (Figure 3D). These cells were Nanosphere ${ }^{\mathrm{TM}}$ fluorescence positive (Figure $3 \mathrm{~A} 1$ and 3D1, respectively), expressed DCX (Figure 3A2 and $3 \mathrm{D} 2$, respectively), and were located either in the lumen or in the walls of brain microvessels (Figure 3A and 3D, respectively).

\section{Expression of the cholinergic neuron marker choline acetyltransferase}

Grafted cells were detected by visualizing the Nanosphere ${ }^{\mathrm{TM}}$ fluorescence (yellow color on Figures 4 and 5). The outcome of the transplantation followed the same pattern as observed in Figures 2 and 3. The highest survival rate in 20-month-old male recipients was obtained after grafting 2-day-old male (Figure 4A) or 20-month-old female NSCs (Figure 4D). Fewer transplanted 20-month-old cells were observed (Figure 4B) as previously described (Figure 2B), and few cells were detected after transplanting 2-day-old female NSCs (Figure 4C). ChAT expression (Figure A2-D2) was found to colocalize with Nanosphere ${ }^{\mathrm{TM}}$ fluorescence (Figure A1-D1). The NSC survival pattern after transplantation in 20-month-old females matched what we previously observed under the same conditions (Figure 3). Many NSCs were present at the transplantation site after grafting 2-day-old female (Figure 5B) or 20-month-old male NSCs (Figure 5C) and expressing the cholinergic phenotype marker ChAT. Fewer cells remained after transplanting 2-day-old male (Figure 5A) and 20-month-old female (Figure 5D) NSCs. ChAT-expressing NSCs were differentiated from endogenous ChAT-positive cells (Figure 5A and 5B, yellow arrow) by the lack of Nanosphere ${ }^{\mathrm{TM}}$ fluorescence in the latter. 




Figure 2 Neural stem cell (NSC) transplantation outcome in 20-month-old male rats. Twenty-month-old male rats were grafted in the left striatum with male or female NSCs isolated from the subventricular zone of either 2-day-old or 20-month-old rats. NSCs were labeled with the fluorescent probe Kodak X-sight 650 (AI-D I, purple). The outcome of the transplantation was then analyzed 8 weeks after the surgical procedure. Immunostaining was performed to specifically detect the neuroblast marker doublecortin (DCX) (A2-D2, green) and the astrocyte marker glial fibrillary acidic protein (A3-D3, red). Cell nuclei were visualized using the fluorophore 4',6-diamidino2-phenylindole. Two-day-old male NSCs (A), 2-day-old female NSCs (B), 20-month-old male NSCs (C), 20-month-old female NSCs (D). (A-D) represent one single confocal plan. The yellow arrows designate Nanosphere ${ }^{\mathrm{TM}}$ fluorescence-positive cells and expressing DCX found in the lumen or wall of brain microvessels. 


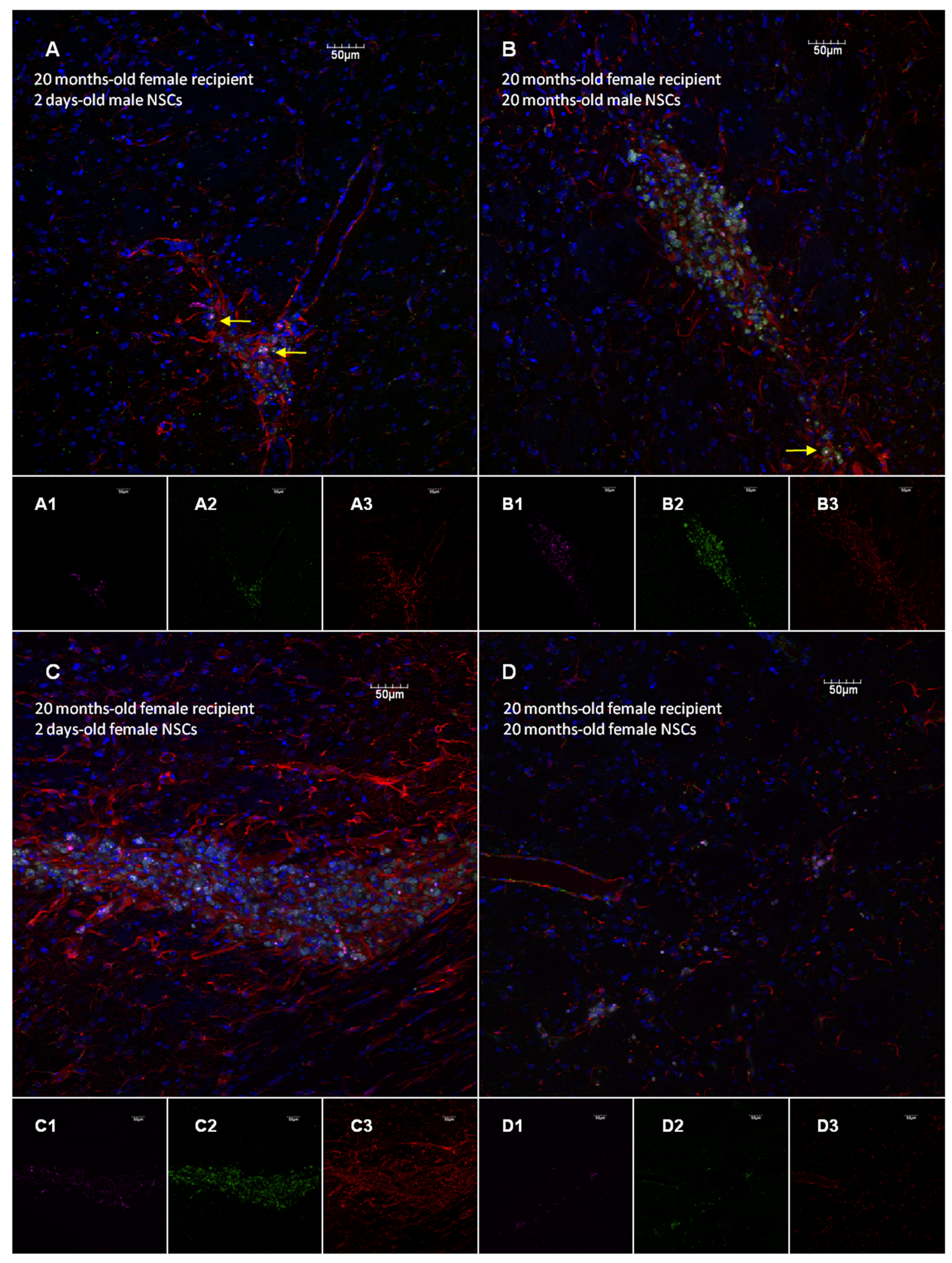

Figure 3 Neural stem cell (NSC) transplantation outcome in 20-month-old female rats. Twenty-month-old female rats were grafted in the left striatum with male or female NSCs isolated from the subventricular zone of either 2-day-old or 20-month-old rats. NSCs were labeled with the fluorescent probe Kodak X-sight 650 (AI-DI). The outcome of the transplantation was then analyzed 8 weeks after the surgical procedure. Immunostaining was performed to specifically detect the neuroblast marker doublecortin (DCX) (A2-D2, green) and the astrocyte marker glial fibrillary acidic protein (A3-D3, red). Cell nuclei were visualized using the fluorophore 4',6-diamidino2-phenylindole. Two-day-old male NSCs (A), 2-day-old female NSCs (B), 20-month-old male NSCs (C), 20-month-old female NSCs (D). (A-D) represent one single confocal plan. The yellow arrows designate Nanosphere ${ }^{\mathrm{TM}}$ fluorescence-positive cells and expressing DCX found in the lumen or wall of brain microvessels. 


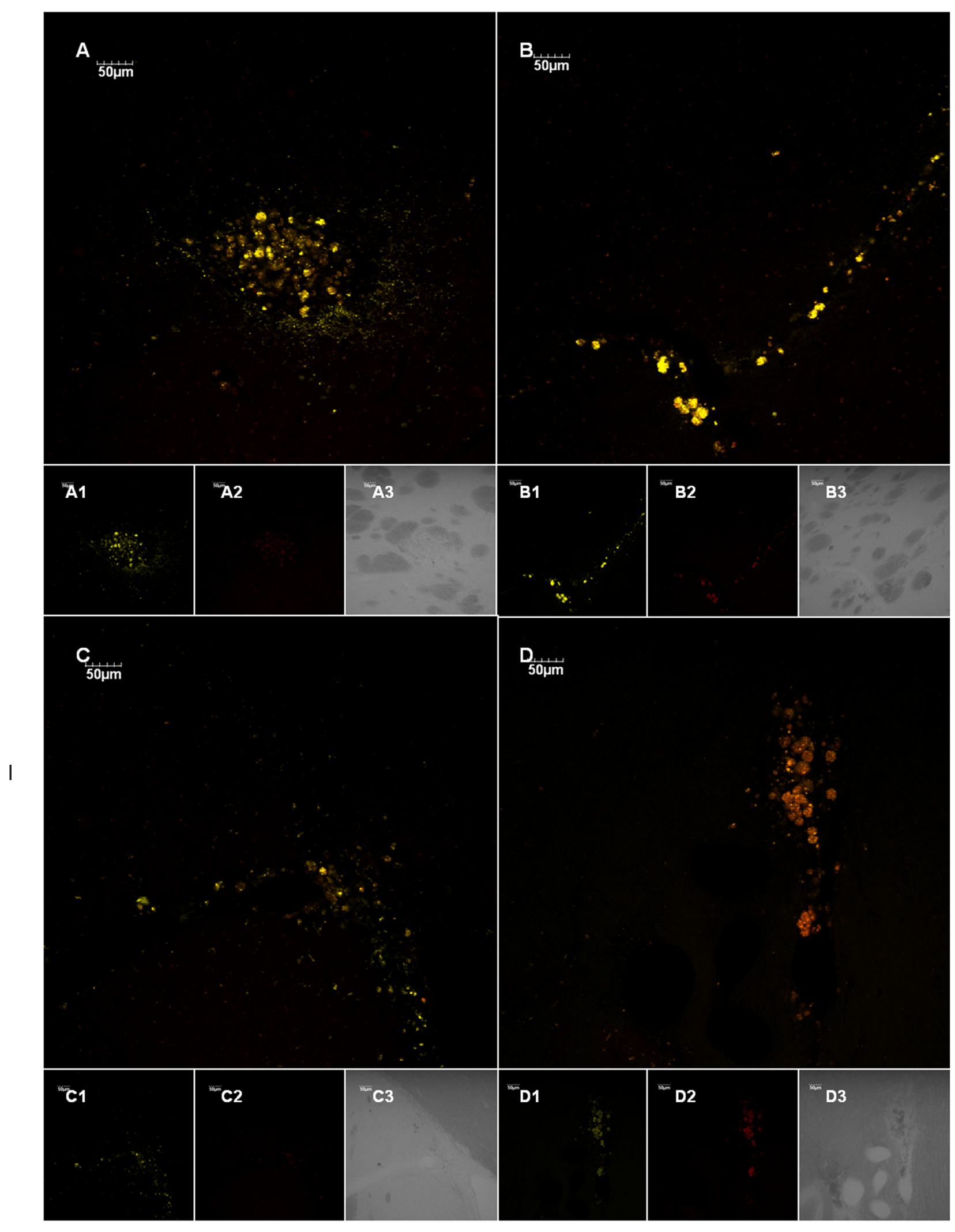

Figure 4 Choline acetyltransferase (ChAT) expression after neural stem cell (NSC) transplantation in 20-month-old male rats. Twenty-month-old male rats were grafted in the left striatum with male or female NSCs isolated from the subventricular zone of either 2-day-old or 20-month-old rats. NSCs were labeled with the fluorescent probe Kodak X-sight 650 (AI-DI, yellow). The expression of ChAT was then analyzed by immunofluorescence 8 weeks after the surgical procedure. Immunostaining was performed to specifically detect ChAT as a marker of neuronal cholinergic phenotype (A2-D2, red). Cell nuclei were visualized using the fluorophore $4^{\prime}, 6$-diamidino-2-phenylindole. Two-day-old male NSCs (A), 2-day-old female NSCs (B), 20-month-old male NSCs (C), 20-month-old female NSCs (D). (A-D) represent one single confocal plan, (A3-D3) phase contrast microscopy, (A-D) the merged image of (AI-DI) and (A2-D2). 


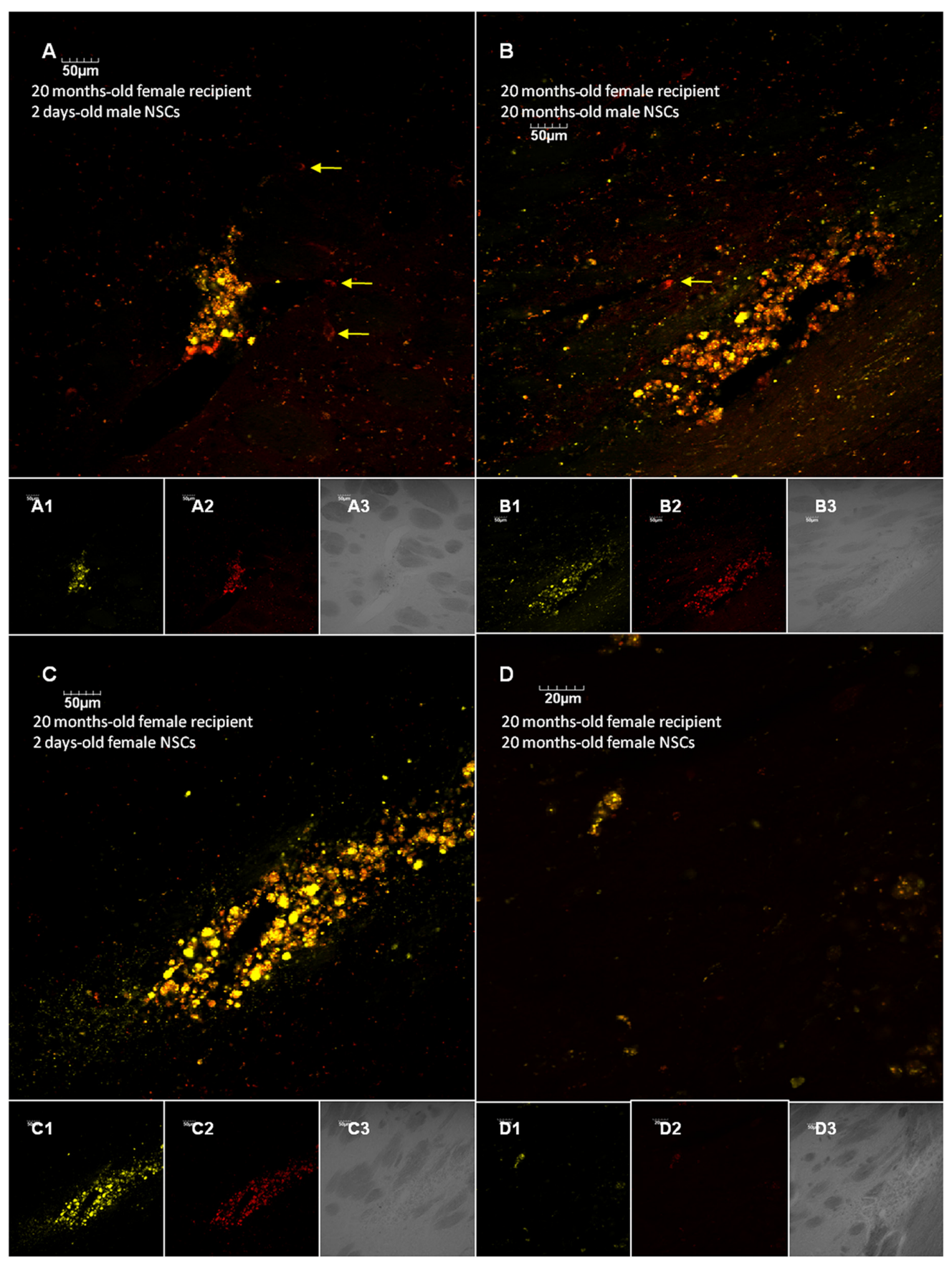

Figure 5 Choline acetyltransferase (ChAT) expression after neural stem cell (NSC) transplantation in 20-month-old female rats. Twenty-month-old female rats were grafted in the left striatum with male or female NSCs isolated from the subventricular zone of either 2-day-old or 20-month-old rats. NSCs were labeled with the fluorescent probe Kodak X-sight 650 (AI-DI, yellow). The expression of ChAT was then analyzed by immunofluorescence 8 weeks after the surgical procedure. Immunostaining was performed to specifically detect ChAT as a marker of neuronal cholinergic phenotype (A2-D2, red). Cell nuclei were visualized using the fluorophore 4',6-diamidino2-phenylindole. Two-day-old male NSCs (A), 2-day-old female NSCs (B), 20-month-old male NSCs (C), 20-month-old female NSCs (D). (A-D) represent one single confocal plan, (A3-D3) phase contrast microscopy, (A-D) the merged image of (AI-DI) and (A2-D2). The yellow arrows designate endogenous ChAT expression unrelated to transplanted NSCs (Kodak X-sight 650 negative).

\section{ChAT endogenous expression in undifferentiated NSCs}

The expression of ChAT was assessed by immunofluorescence in male and female NSCs grown as neurospheres in order to determine whether the expression of ChAT detected in transplantated NSCs revealed the acquisition of a neural phenotype or was already occurring in NSCs prior to the transplantation. ChAT expression was not detected in male (Figure 6A) or female (Figure 6B) NSCs. As shown on the color scale, the immunofluorescence 

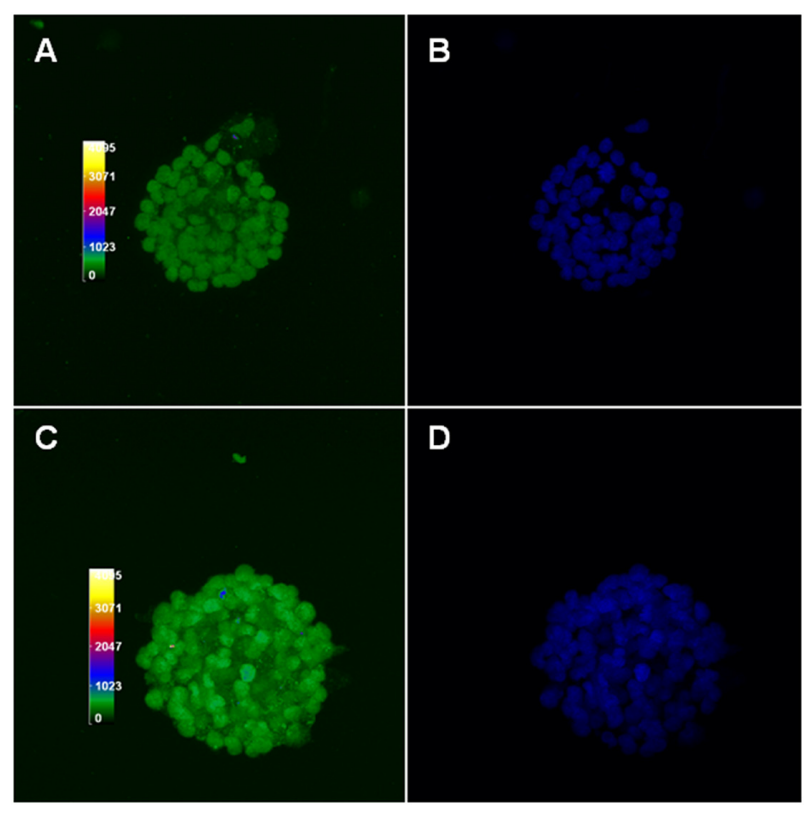

Figure 6 Assessment of choline acetyltransferase (ChAT) expression in undifferentiated male and female neural stem cells (NSCs). Male (A, B) and female (C, D) NSCs were grown as neurospheres and assessed for ChAT expression by immunofluorescence. No detectable ChAT expression level was observed in male (A) or female (B) NSCs, as shown by the fluorescence emission corresponding to background noise level. Cell nuclei were visualized using the fluorophore 4',6-diamidino-2-phenylindole (B, D).

for ChAT expression was equivalent to the background signal.

\section{Discussion}

We previously demonstrated in vitro that male and female NSCs exhibit intrinsic biologic differences, sex and age being two major determinants of the phenotypic fate, followed by NSCs in the process of differentiation. ${ }^{1,2}$ We showed that NSCs isolated from the SVZ of 3-month-old male rats were mainly expressing neuronal markers when differentiated with retinoic acid, whereas female NSCs were essentially following an astroglial fate. These phenotypic fates were altered with aging in a manner that was dependent on the cell sex, as shown in NSCs isolated from 20-month-old animals. Such sex-based differences in NSC neurogenic properties were related to a differential expression of $E R \alpha$ and $E R \beta^{1}$ and aromatase, ${ }^{2}$ the enzyme that converts testosterone in estradiol. ${ }^{10}$ Herein we provided evidence that NSC sex-based differences across ages initially observed in vitro translate into in vivo differential outcomes after transplantation into rat brains.

NSC transplantation in the striatum of 2-day-old pups overall resulted in an increased endogenous neurogenesis, suggesting that NSCs may contribute to brain repair not necessarily by replacing lost cells but rather by triggering in situ regeneration. Interestingly, the effect of NSC transplantation on endogenous neurogenesis has been reported by two recent studies in two different animal models. Indeed, in one case, murine NSCs improved a triple transgenic mouse model of Alzheimer's disease ( $3 \times \mathrm{Tg}$-AD ${ }^{\text {APP-PS1-Tau }}$ ) cognitive performance through BDNF synthesis, ${ }^{11}$ and others reported that grating human NSCs enhanced endogenous axonal sprouting through modification of glial response in a rat model of neonatal brain ischemia. ${ }^{12}$ Strikingly, none of the grafted NSCs in the brain of 2-day-old pups was detected 8 weeks after transplantation at the time we observed an increase of the endogenous neurogenesis. A reason may lay in the developmental dynamic of an immature 2-day-old brain where the cell turnover is still high, leading to the quick elimination of the grafted cells as the brain is maturing, unlike what we observed in the adult rat brains. Cognitive improvement without long-term detection of the grafted NSCs has been previously reported; indeed, other authors have shown that NSCs isolated from mouse SVZ and grafted in rat hippocampus resulted in cognitive impairment and behavior alteration induced by granule cell lesion. ${ }^{13}$ However, these authors were unable to detect any of the grafted NSCs several months after the transplantation. These results led them to conclude that the beneficial effect of grafted NSCs on the animals' performances were unlikely to be due to cell replacement but rather to induced endogenous neurogenesis and synaptogenesis. ${ }^{13}$ Furthermore, it appeared that the graft-induced stimulation of endogenous neurogenesis highly dependent on the sex of the recipient animal and the sex and age of the NSC donor. Indeed, grafting NSCs isolated from 2-day-old male rats resulted in both males and females in the formation of humps in the ependymal layer protruding inside the ipsilateral ventricle and containing many nestin-positive and DCX-positive cells. Transplanting 2-day-old female NSCs, on the other hand, induced homogenous thickening of the subependymal layer and a significant increase of nestin and DCX expression. However, these histological alterations appeared to be more dramatic in male recipients than in female recipients. On the other hand, grafting NSCs isolated from old rats, male and female, in male pups resulted in both cases in the formation in the ipsilateral ependymal of many clusters of cells expressing nestin and DCX, whereas only the transplantation of male old NSCs provided significant results in female pups with an enlargement of the subependymal area. This enlargement, probably due to the presence of a higher number of progenitor cells, 
displayed a dramatic increase of nestin and DCX expression, although not as important as when 2-day-old female NSCs were grafted in male recipients. Although there is no obvious reason to explain such discrepancy, these results suggest that NSC intrinsic properties and environmental pharmacologic pressure, ie, steroids, may play an important role.

We recently showed that NSCs displayed a sexual dimorphism in the expression of ER $\alpha / \beta^{1}$ and aromatase (CYP19), ${ }^{2}$ the enzyme that converts testosterone in estrogen. CYP19 expression level in female NSCs was at the detection limit level, whereas male NSC expression was significant. We hypothesized that such dimorphism may account for the sexual difference in the neural fate of differentiating NSCs we observed in vitro. ${ }^{1,2}$ A paracrine mechanism originating from transplanted stem cells has previously been evoked to explain increased SVZ neurogenesis and functional recovery in a rat model of cerebral ischemia without apparent integration of the transplanted stem cells in the niche structure. ${ }^{14}$ Others showed that 14 days after having transplanted adipose tissue-derived stem cells in an artificial nerve conduit in a rat model of sciatic nerve injury, no viable transplanted cells were detectable, although axonal outgrowth was stimulated and greater Schwann cell proliferation was evoked in the distal pump. ${ }^{15}$ The authors concluded that the effect was probably due to a growth that remains to be determined and the diffusion of which was triggered by the transplanted stem cells.

Unlike what we observed in the 2-day old pups, grafted NSCs were still present in the adult brain 8 weeks after the transplantation. Grafted cells were clearly visible at the transplantation site, as previously described by other authors. ${ }^{16-19}$ Dramatic differences in cell survival at the transplantation site were observed depending on the sex of the recipient and the sex and age of the donor. Indeed, very few cells were observed in male recipients after transplanting NSCs isolated from 20-month-old males or 2-day-old females, whereas survival rate at 8 weeks was extremely high in the case of 2-day-old male and 20-month-old female donors. Interestingly, the survival pattern was exactly reversed in male compared with female recipients, suggesting that the effect of the hormonal impregnation on the transplantation outcome is significant but is not the only factor. Indeed, the survival of the grafted NSCs greatly differed depending on the sex and age of the donor, regardless of the sex of the recipients, leading to the hypothesis that the manner NSCs interact with their new environment may largely depend on factors that are specific to their sex-like
ER or aromatase expression. ${ }^{1,2}$ Sexual dimorphism has been previously described for muscle-derived stem cells for which the females have been shown to generate functional muscle or bone tissue and in a larger amount and faster than their male counterparts. ${ }^{3,6}$ Such observation has also been made when mesenchymal stem cells (MSCs) were used to repair infracted myocardium when female MSC grafting resulted in a higher recovery of the cardiac function than with the male MSCs. ${ }^{5,20}$ In successful transplantation combinations, few cells were seen at a short distance from the injection site, suggesting that some of the cells may have entered a migration process. At 8 weeks, many transplanted NSCs were expressing the neuroblast marker DCX, suggesting a differentiation toward the neuronal fate. Many cells were expressing the astrocyte marker GFAP, supporting a differentiation process toward astroglial fate. Few cells were also found to be coexpressing DCX and GFAP, suggesting that these particular NSCs were still at a very early stage of the differentiation process and at the crossroads between astroglial and neuronal fate. The morphology of the transplanted cells and their neural marker expression pattern were quite similar to what was reported by other authors after transplantation of human cord blood-derived stem cells in rat brains. ${ }^{21,22}$ Indeed, most of the grafted cells remained at the injection site, with few cells found to be migrating. Interestingly, many transplanted cells that were recognizable because of a Nanosphere ${ }^{\mathrm{TM}}$ fluorescence-positive signal were observed in the wall of brain microvessels or in the lumen in direct contact with the endothelium. The reason underlying such a finding remains to be established. However, pericytes embryologically derive from neural crest, ${ }^{23,24}$ which may signify that some of the transplanted NSCs have been oriented toward an angiogenic process rather than toward a neurogenic one.

Many of the grafted cells were expressing ChAT 8 weeks after transplantation. No evidence of ChAT expression was found in undifferentiated NSCs, suggesting that NSCs acquired a cholinergic neuronal phenotype following their transplantation into the striatum. Interestingly, transplanted NSCs were reported to find cues in the area of the brain, leading them to acquire region-specific phenotype. ${ }^{25}$ The presence of endogenous cholinergic neurons in the vicinity of the transplantation site may support the hypothesis according to which these resident neurons may have guided the differentiation of the transplanted NSCs. ChAT expression was observed in grafted cells, even when few cells were found to have 
survived the transplantation; this suggests that although the factors sex and age regulated the NSC survival, they did not have any effect on ChAT expression.

\section{Conclusion}

In previous work, we demonstrated that NSCs derived from males and females follow different neural fates in vitro in response to all-transretinoic acid treatment, with male NSCs expressing mainly neuron-specific markers as compared with female NSCs expressing astroglial markers. ${ }^{1,2}$ In addition, we found that age-matched male and female undifferentiated NSCs express different levels of steroid receptors, in particular ER $\alpha$ and ER $\beta,{ }^{1}$ and aromatase (CYP19), ${ }^{2}$ the enzyme that converts testosterone in estradiol. Remarkably, the results presented herein suggest that the NSC sexual dimorphism we previously demonstrated in vitro translated in vivo in NSC transplantation. In particular, the outcome of the NSC transplantation was highly dependent on the sex and age of the combination donor/recipient. Stem cell therapy is a promising approach for brain repair strategy. However, "know-how" is extremely limited, and the clinical trials based on stem cell engraftment in the human brain have so far obtained only an anecdotal success rate. A possible explanation may be attributable to the lack of matching specificity between the donor and the receiver, which, for the NSCs, might go beyond the sole lack of immunocompatibility. Data generated from our work may allow us in the future to answer the question "What NSCs and for whom?" and consequently lead to the optimization of the grafting process and improvement of the clinical prognosis.

\section{Acknowledgment}

This work has been funded by McGill University Health Centre start-up funds, Royal Victoria Hospital Award, and the Canadian Institutes of Health Research catalyst grant IAP 92827.

\section{Disclosure}

The authors report no conflicts of interest in this work.

\section{References}

1. Waldron J, McCourty AM, Lecanu L. Aging differentially affects male and female neural stem cell neurogenic properties. Stem Cells Cloning Adv Appl. 2010;3119-3127.

2. Waldron J, McCourty AM, Lecanu L. Neural stem cell sex dimorphism in aromatase (CYP19) expression: a basis for differential neural fate. Stem Cells Cloning Adv Appl. 2011;3:175-182.
3. Deasy BM, Lu A, Tebbets JC, et al. A role for cell sex in stem cell-mediated skeletal muscle regeneration: female cells have higher muscle regeneration efficiency. J Cell Biol. 2007;177:73-86.

4. Deasy BM, Schugar RC, Huard J. Sex differences in muscle-derived stem cells and skeletal muscle. Crit Rev Eukaryot Gene Expr. 2008;18: 173-188.

5. Herrmann JL, Abarbanell AM, Weil BR, et al. Gender dimorphisms in progenitor and stem cell function in cardiovascular disease. J Cardiovasc Transl Res. 2010;3:103-113.

6. Matsumoto T, Kubo S, Meszaros LB, et al. The influence of sex on the chondrogenic potential of muscle-derived stem cells: implications for cartilage regeneration and repair. Arthritis Rheum. 2008;58: 3809-3819.

7. Yuan J, Yu JX, Ge J. Sexual dimorphism on the neurogenic potential of rhesus monkeys mesenchymal stem cells. Biochem Biophys Res Commun. 2010;396:394-400.

8. Laywell ED, Kukekov VG, Suslov O, et al. Production and analysis of neurospheres from acutely dissociated and postmortem CNS specimens. Methods Mol Biol. 2002;198:15-27.

9. Richardson RM, Holloway KL, Bullock MR, et al. Isolation of neuronal progenitor cells from the adult human neocortex. Acta Neurochir (Wien). 2006;148:773-777.

10. Selmanoff MK, Brodkin LD, Weiner RI, et al. Aromatization and 5alpha-reduction of androgens in discrete hypothalamic and limbic regions of the male and female rat. Endocrinology. 1977;101: 841-848.

11. Blurton-Jones M, Kitazawa M, Martinez-Coria H, et al. Neural stem cells improve cognition via BDNF in a transgenic model of Alzheimer disease. Proc Natl Acad Sci U S A. 2009;106:13594-13599.

12. Daadi MM, Davis AS, Arac A, et al. Human neural stem cell grafts modify microglial response and enhance axonal sprouting in neonatal hypoxic-ischemic brain injury. Stroke. 2010;41:516-523.

13. Jeltsch H, Yee J, Aloy E, et al. Transplantation of neurospheres after granule cell lesions in rats: cognitive improvements despite no longterm immunodetection of grafted cells. Behav Brain Res. 2003;143: 177-191.

14. Stroemer P, Patel S, Hope A, et al. The neural stem cell line CTX0E03 promotes behavioral recovery and endogenous neurogenesis after experimental stroke in a dose-dependent fashion. Neurorehabil Neural Repair. 2009;23:895-909.

15. Erba P, Mantovani C, Kalbermatten DF, et al. Regeneration potential and survival of transplanted undifferentiated adipose tissue-derived stem cells in peripheral nerve conduits. J Plast Reconstr Aesthet Surg. 2010;63:e811-e817.

16. Capetian P, Knoth R, Maciaczyk J, et al. Histological findings on fetal striatal grafts in a Huntington's disease patient early after transplantation. Neuroscience. 2009;160:661-675.

17. Darsalia V, Kallur T, Kokaia Z. Survival, migration and neuronal differentiation of human fetal striatal and cortical neural stem cells grafted in stroke-damaged rat striatum. Eur J Neurosci. 2007;26: 605-614.

18. Li JM, Zhu H, Lu S, et al. Migration and differentiation of human mesenchymal stem cells in the normal rat brain. Neurol Res. 2010;3: $84-92$.

19. Zhu Q, Ma J, Yu L, et al. Grafted neural stem cells migrate to substantia nigra and improve behavior in Parkinsonian rats. Neurosci Lett. 2009;462:213-218.

20. Herrmann JL, Abarbanell AM, Weil BR, et al. Optimizing stem cell function for the treatment of ischemic heart disease. J Surg Res. 2011;166:138-145.

21. Jablonska A, Kozlowska H, Markiewicz I, et al. Transplantation of neural stem cells derived from human cord blood to the brain of adult and neonatal rats. Acta Neurobiol Exp (Wars). 2010;70:337-350.

22. Kozlowska H, Jablonka J, Janowski M, et al. Transplantation of a novel human cord blood-derived neural-like stem cell line in a rat model of cortical infarct. Stem Cells Dev. 2007;16:481-488. 
23. Etchevers HC, Vincent C, Le Douarin NM, et al. The cephalic neural crest provides pericytes and smooth muscle cells to all blood vessels of the face and forebrain. Development. 2001;128:1059-1068.

24. Zachariah MA, Cyster JG. Neural crest-derived pericytes promote egress of mature thymocytes at the corticomedullary junction. Science. 2010;328:1129-1135.
25. Yang M, Stull ND, Berk MA, et al. Neural stem cells spontaneously express dopaminergic traits after transplantation into the intact or 6-hydroxydopamine-lesioned rat. Exp Neurol. 2002;177:50-60.

\section{Publish your work in this journal}

Stem Cells and Cloning: Advances and Applications is an international, peer-reviewed, open access journal. Areas of interest in stem cell research include: Embryonic cell stems; Adult stem cells; Blastocysts; Cordblood stem cells; Stem cell transformation and culture; Therapeutic cloning; Umbilical cord blood and bone marrow cells; Laboratory, animal and human therapeutic studies; Philosophical and ethical issues related to stem cell research. This journal is indexed on CAS. The manuscript management system is completely online and includes a quick and fair peer-review system. Visit http://www.dovepress.com/ testimonials.php to read real quotes from published authors.

\footnotetext{
Submit your manuscript here: http://www.dovepress.com/stem-cells-and-cloning-advances-and-applications-journal
} 